\title{
Effects Of Heat Source And Thermal Diffusion On An Unsteady Free Convection Flow Along A Porous Plate With Constant Heat And Mass Flux In A Rotating System Under Slip Boundary Condition
}

\author{
Hoshiyar Singh ${ }^{1}$, N.C. Jain ${ }^{2}$ and D. Chaudhary ${ }^{3}$ \\ ${ }^{1}$ Department of Mathematics, Jaipur National University Jagatpura, Jaipur-302025, Rajasthan (India) \\ ${ }^{2}$ Department of Mathematics, University of Rajasthan, Jaipur-302055, Rajasthan (India)
}

\begin{abstract}
The present analysis is made to investigate the effects of heat source and thermal diffusion on an unsteady free convection flow along a porous vertical plate in a rotating system. The plate is subjected to constant heat and mass flux also. The problem is solved analytically and expressions for velocity. Energy and temperature profiles, skin friction and Nusselt number are obtained. The effects of different parameter entered in the problem are discussed on the primary and secondary velocities, temperature and concentration distributions, primary and secondary skin frictions and Nusselt number with the help of tables and graphs.
\end{abstract}

Key Words: Diffusion, Heat Source, Porous Medium, Slip Velocity, Unsteady Flow.

\section{Introduction}

The flow through a porous medium is a common occurrence in industrial environment and so the heat transfer problems of viscous incompressible fluid through a porous medium has attracted the interest of many research workers in view of its applications in geophysics, astrophysics, aerodynamics, boundary layer control and so on.

A number of authors such as Soundalgekar et al. [1], Raptis and Perdikis [2], Mohammad [3], Helmy [4] and recently Jain and Gupta [5] have investigated flow through porous medium on different geometries. The effect of variable permeability on combined free and forced convection in porous media was studied by Chandrasekhara and Namboodiri [6] and Mixed Convection in porous media with uniform heat flux on vertical surface was studied by Joshi and Gebhart [7]. Lai and Kulacki [8] studied the effect of variable viscosity along a vertical surface in a saturated porous medium.

The phenomena of heat and mass transfer is encountered in chemical process industries such as food processing and polymer products. Raptis and Kafousias [9], Bejan and Khair [10], Elbashbeshy [11], Acharya et al. [12], Chand et al. [13] are some of the workers to study the different problems through porous medium with mass transfer effects.

In high attitude flights, situation may arise when the flow becomes unsteady and slip at the boundary take place as well. In such situation of slip flow ordinary continuum approach fails to yield satisfactory result [Tsien [14], Street [15]]. Many authors have solved problems taking slip conditions at the boundary. Recently Jothimani and Anjali Devi [16], Jain et al. [17] and Gupta et al. [18] have considered slip boundary conditions in their problems.

In the present work we study the effects of heat source and permeability on unsteady free convection rotating flow of a viscous fluid past a porous vertical plate with time dependent suction and velocity slip boundary conditions. At the plate there is constant heat flux and mass flux. Perturbation technique is used to obtain the expressions of velocity and temperature fields, concentration profile, skin friction and Nusselt number. Effects of different parameters viz. Grashoff number of heat transfer, Grashoff number for mass transfer, slip parameter, permeability parameter, heat source parameter, Diffusion parameter and Schmidt number are discussed and shown graphically. It is observed that increase in Schmidt number decreases primary skin friction $\left(\tau_{\mathrm{p}}\right)$ as well as secondary skin friction $\left(\tau_{\mathrm{s}}\right)$ while increase in Grashoff number increase both the skin fictions. Moreover, increase in Prandtl number decreases Nusselt Number $(\mathrm{Nu})$.

\section{Mathematical Formulation}

We consider an unsteady free convection flow of an incompressible viscous fluid through a porous medium past an infinite vertical porous plate with constant heat and mass fluxes. Let both the fluid and plate be in a state of rigid rotation with uniform angular velocity $\Omega$ about z-axis taken normal to the plane and plate is taken electrically non-conducting. Moreover the plate is assumed to coincide with the plane $\mathrm{z}=0$. As the plate is infinite in extent and the flow is unsteady, all the physical variables depend on $\mathrm{z}$ and $\mathrm{t}$ only. 
For the governing equations for a free convective flow with heat source along a porous vertical plate in rotating system are given as:

\section{Continuity equation}

$$
\frac{\partial \mathrm{w}}{\partial \mathrm{z}}=0
$$

\section{Momentum equations}

$$
\begin{gathered}
\frac{\partial \mathrm{u}}{\partial \mathrm{t}}+\mathrm{w} \frac{\partial \mathrm{u}}{\partial \mathrm{z}}-2 \Omega \mathrm{v}=\mathrm{v} \frac{\partial^{2} \mathrm{u}}{\partial \mathrm{z}^{2}}+\mathrm{g} \beta\left(\mathrm{T}-\mathrm{T}_{\infty}\right)+\mathrm{g} \beta^{\prime}\left(\mathrm{C}-\mathrm{C}_{\infty}\right)-\frac{v}{\mathrm{~K}} \mathrm{u} \\
\frac{\partial \mathrm{v}}{\partial \mathrm{t}}+\mathrm{w} \frac{\partial \mathrm{v}}{\partial \mathrm{z}}+2 \Omega \mathrm{u}=\mathrm{v} \frac{\partial^{2} \mathrm{v}}{\partial \mathrm{z}^{2}}-\frac{v}{\mathrm{~K}} \mathrm{v}
\end{gathered}
$$

Energy equation

$$
\frac{\partial \mathrm{T}}{\partial \mathrm{t}}+\mathrm{w} \frac{\partial \mathrm{T}}{\partial \mathrm{z}}=\frac{\lambda}{\rho \mathrm{C}_{\mathrm{p}}} \frac{\partial^{2} \mathrm{~T}}{\partial \mathrm{z}^{2}}+\frac{\mathrm{S}^{*}}{\rho \mathrm{C}_{\mathrm{p}}}\left(\mathrm{T}-\mathrm{T}_{\infty}\right)
$$

\section{Concentration equation}

$$
\frac{\partial \mathrm{C}}{\partial \mathrm{t}}+\mathrm{w} \frac{\partial \mathrm{C}}{\partial \mathrm{z}}=\mathrm{D} \frac{\partial^{2} \mathrm{C}}{\partial \mathrm{z}^{2}}+\mathrm{D}_{\ell} \frac{\partial^{2} \mathrm{~T}}{\partial \mathrm{z}^{2}}
$$

Here $\rho$ is the density, $g$ is the acceleration due to gravity, $\beta$ is the coefficient of volume expansion, $\beta^{\prime}$ is the coefficient of concentration expansion, $v$ is the kinematic viscosity, $\mathrm{T}_{\infty}$ is the temperature of the fluid in the free stream, $\mathrm{C}_{\infty}$ is the concentration at infinite, $\mathrm{u}$ and $\mathrm{v}$ are the velocities in $\mathrm{x}$ and $\mathrm{y}$ direction respectively, $\mathrm{T}$ is the temperature of the fluid, $\mathrm{K}$ is the permeability of porous medium, $\lambda$ is the thermal conductivity, $\mathrm{D}$ is the concentration diffusivity, $C_{p}$ is the specific heat at constant pressure, $S^{*}$ is the coefficient of heat source, $D_{\ell}$ is coefficient of diffusivity.

The boundary conditions are:

$$
\left.\begin{array}{l}
\mathrm{u}=\mathrm{L} \frac{\partial \mathrm{u}}{\partial \mathrm{z}}, \mathrm{v}=\mathrm{L} \frac{\partial \mathrm{v}}{\partial \mathrm{z}}, \frac{\partial \mathrm{T}}{\partial \mathrm{z}}=-\frac{\mathrm{q}^{\prime}}{\lambda}, \frac{\partial \mathrm{C}}{\partial \mathrm{z}}=-\frac{\mathrm{m}^{\prime}}{\mathrm{D}} \quad \text { at } \mathrm{z}=0 \\
\mathrm{u} \rightarrow 0, \mathrm{v} \rightarrow 0, \mathrm{~T} \rightarrow \mathrm{T}_{\infty}, \mathrm{C} \rightarrow \mathrm{C}_{\infty} \quad \text { as } \mathrm{z} \rightarrow \infty
\end{array}\right\}
$$

Here q' and m' are uniform heat and concentration flux at the plate respectively, $\mathrm{L}$ being Mean free path. Integration of equation (2.1) gives:

$$
\mathrm{w}=-\mathrm{w}_{0}\left(1+\in \mathrm{e}^{-\mathrm{nt}}\right)
$$

Using equation (2.7) and introducing following non-dimensional quantities

$$
\begin{aligned}
& \mathrm{u}^{*}=\frac{\mathrm{u}}{\mathrm{w}_{0}}, \mathrm{v}^{*}=\frac{\mathrm{v}}{\mathrm{w}_{0}}, \mathrm{z}^{*}=\frac{\mathrm{zw}_{0}}{v}, \mathrm{n}^{*}=\frac{\mathrm{m}}{\mathrm{w}_{0}^{2}}, \mathrm{t}^{*}=\frac{\mathrm{w}_{0}^{2} \mathrm{t}}{v} \\
& \theta=\frac{\left(\mathrm{T}-\mathrm{T}_{\infty}\right) \mathrm{w}_{0} \lambda}{\mathrm{q}^{\prime} \mathrm{v}}, \phi=\frac{\left(\mathrm{C}-\mathrm{C}_{\infty}\right) \mathrm{w}_{0} \mathrm{D}}{\mathrm{m}^{\prime} \mathrm{v}}, \mathrm{K}^{*}=\frac{\mathrm{K} \mathrm{\textrm {w } _ { 0 } ^ { 2 }}}{\mathrm{v}^{2}} \text { (Permeability parameter) } \\
& \operatorname{Pr}=\frac{\mu \mathrm{C}_{\mathrm{p}}}{\lambda} \text { (Prandtl number), } \mathrm{Sc}=\frac{\mathrm{v}}{\mathrm{D}} \text { (Schimdt number) } \\
& \mathrm{E}=\frac{\mathrm{v} \Omega}{\mathrm{w}_{0}^{2}} \text { (Rotation velocity parameter), } \mathrm{S}^{*}=\frac{\mathrm{v}^{2} \mathrm{~S}}{\lambda \mathrm{w}_{0}^{2}} \text { (Heat Source parameter) }
\end{aligned}
$$


$\mathrm{Gr}=\frac{\mathrm{g} \beta \mathrm{q}^{\prime} v^{2}}{\mathrm{w}_{0}^{4} \lambda}$ (Grashoff number for heat transfer $)$,

$\mathrm{Gm}=\frac{\mathrm{g} \beta^{\prime} \mathrm{m}^{\prime} v^{2}}{\mathrm{w}_{0}^{4} \mathrm{D}}$ (Grashoff number for mass transfer $)$

$\mathrm{A}=\frac{\mathrm{q}^{\prime} \mathrm{D}_{\ell}}{\lambda \mathrm{m}^{\prime}}$ (Diffusion parameter), $\mathrm{h}=\frac{\mathrm{L} \mathrm{w}_{0}}{\mathrm{v}}$ (slip parameter)

Equations (2.2) to (2.5) reduce to the following form after dropping the asterisks over them:

$$
\begin{aligned}
& \frac{\partial^{2} \mathrm{q}}{\partial \mathrm{z}^{2}}+\left(1+\in \mathrm{e}^{-\mathrm{nt}}\right) \frac{\partial \mathrm{q}}{\partial \mathrm{z}}-\frac{\partial \mathrm{q}}{\partial \mathrm{t}}-\left(\mathrm{s}+\frac{1}{\mathrm{~K}}\right)=-\mathrm{Gr} \theta-\mathrm{Gm} \phi \\
& \frac{\partial^{2} \theta}{\partial \mathrm{z}^{2}}+\operatorname{Pr}\left(1+\in \mathrm{e}^{-\mathrm{nt}}\right) \frac{\partial \theta}{\partial \mathrm{z}}-\operatorname{Pr} \frac{\partial \theta}{\partial \mathrm{t}}=-\mathrm{S} \theta \\
& \frac{\partial^{2} \phi}{\partial \mathrm{z}^{2}}+\operatorname{Sc}\left(1+\in \mathrm{e}^{-\mathrm{nt}}\right) \frac{\partial \phi}{\partial \mathrm{z}}-\mathrm{Sc} \frac{\partial \phi}{\partial \mathrm{t}}=-\mathrm{A} \frac{\partial^{2} \theta}{\partial \mathrm{z}^{2}}
\end{aligned}
$$

where $\mathrm{q}=\mathrm{u}+\mathrm{iv}$ and $\mathrm{s}=2 \mathrm{iE}$.

The corresponding boundary conditions become:

$$
\left.\begin{array}{ll}
\mathrm{q}=\mathrm{h} \frac{\partial \mathrm{q}}{\partial \mathrm{z}}, \frac{\partial \theta}{\partial \mathrm{z}}=-1, \frac{\partial \phi}{\partial \mathrm{z}}=-1 & \text { at } \mathrm{z}=0 \\
\mathrm{q} \rightarrow 0, \theta \rightarrow 0, \phi \rightarrow 0 & \text { as } \mathrm{z} \rightarrow \infty
\end{array}\right\}
$$

\section{Solution Of The Problem}

To solve equations (2.9) to (2.11), we follow the perturbation technique in the form $(\in<<1)$

$$
\begin{aligned}
& \mathrm{q}=\mathrm{q}_{1}(\mathrm{z})+\in \mathrm{e}^{-\mathrm{nt}} \mathrm{q}_{2}(\mathrm{z})+0\left(\in^{2}\right) \\
& \theta=\theta_{1}(\mathrm{z})+\in \mathrm{e}^{-\mathrm{nt}} \theta_{2}(\mathrm{z})+0\left(\in^{2}\right) \\
& \phi=\phi_{1}(\mathrm{z})+\in \mathrm{e}^{-\mathrm{nt}} \phi_{2}(\mathrm{z})+0\left(\in^{3}\right)
\end{aligned}
$$

Substitution of Equations (3.1) to (3.3) in Equations (2.9) to (2.11) and equating the coefficient of like powers of $\in$ (neglecting $\epsilon^{2}$ etc.), we obtain the following set of differential equations

$$
\begin{aligned}
& \frac{\mathrm{d}^{2} \mathrm{q}_{1}}{\mathrm{dz}^{2}}+\frac{\mathrm{dq}_{1}}{\mathrm{dz}}-\left[\mathrm{s}+\frac{1}{\mathrm{k}}\right] \mathrm{q}_{1}=-\mathrm{Gr} \theta_{1}-\mathrm{Gm} \phi_{1} \\
& \frac{\mathrm{d}^{2} \mathrm{q}_{2}}{\mathrm{dz}^{2}}+\frac{\mathrm{dq}_{1}}{\mathrm{dz}}-\left[\mathrm{n}+\mathrm{s}+\frac{1}{\mathrm{~K}}\right] \mathrm{q}_{2}=- \text { Gr } \theta_{1}-\mathrm{Gm} \phi_{2}-\frac{\mathrm{dq}_{1}}{\mathrm{dz}} \\
& \frac{\mathrm{d}^{2} \theta_{1}}{\mathrm{dz}^{2}}+\operatorname{Pr} \frac{\mathrm{d} \theta_{1}}{\mathrm{dz}}=-\mathrm{S} \theta_{1} \\
& \frac{\mathrm{d}^{2} \theta_{2}}{\mathrm{dz}^{2}}+\operatorname{Pr} \frac{\mathrm{d} \theta_{2}}{\mathrm{dz}}=-(\mathrm{n} \operatorname{Pr}+\mathrm{S}) \theta_{2}-\operatorname{Pr} \frac{\mathrm{d} \theta_{1}}{\mathrm{dz}}
\end{aligned}
$$




$$
\begin{aligned}
& \frac{\mathrm{d}^{2} \phi_{1}}{\mathrm{dz}^{2}}+\mathrm{Sc} \frac{\mathrm{d} \phi_{1}}{\mathrm{dz}}+\mathrm{A} \frac{\mathrm{d}^{2} \theta_{1}}{\mathrm{dz}^{2}}=0 \\
& \frac{\mathrm{d}^{2} \phi_{2}}{\mathrm{dz}}+\mathrm{Sc} \frac{\mathrm{d} \phi_{2}}{\mathrm{dz}}=-\mathrm{n} \operatorname{Sc} \phi_{2}-\mathrm{Sc} \frac{\mathrm{d} \phi_{1}}{\mathrm{dz}}-\mathrm{A} \frac{\mathrm{d}^{2} \theta_{2}}{\mathrm{dz}^{2}}
\end{aligned}
$$

the corresponding boundary conditions are:

$$
\left.\begin{array}{l}
\mathrm{q}_{1}=\mathrm{h} \frac{\mathrm{dq} \mathrm{q}_{1}}{\mathrm{dz}}, \mathrm{q}_{2}=\mathrm{h} \frac{\mathrm{dq} \mathrm{q}_{2}}{\mathrm{dz}} ; \frac{\mathrm{d} \theta_{1}}{\mathrm{dz}}=-1, \frac{\mathrm{d} \theta_{2}}{\mathrm{dz}}=0 ; \frac{\mathrm{d} \phi_{1}}{\mathrm{dz}}=-1, \frac{\mathrm{d} \phi_{2}}{\mathrm{dz}}=0 \text { at } \mathrm{z}=0 \\
\mathrm{q}_{1} \rightarrow 0, \mathrm{q}_{2} \rightarrow 0 ; \theta_{1} \rightarrow 0, \theta_{2} \rightarrow 0 ; \phi_{1} \rightarrow 0, \phi_{2} \rightarrow 0 \quad \text { as } \mathrm{z} \rightarrow \infty
\end{array}\right\}
$$

On solving equations (3.4) to (3.9) under the transformed boundary conditions (3.10), we get the solution for $\theta, \phi$ and $\mathrm{q}$ as follows

$$
\begin{gathered}
\theta=\frac{1}{D_{1}} e^{-D_{1} z}+\in e^{-n t}\left(R_{1} e^{-D_{1} z}+R_{2} e^{-D_{2} z}\right) \\
\phi=R_{3} e^{-S c z}-R_{4} e^{-D_{1} z}+\in e^{-n t}\left(R_{5} e^{-S c z}-R_{6} e^{-D_{1} z}-R_{7} e^{-D_{2} z}+R_{8} e^{-D_{3} z}\right) \\
q=-R_{9} e^{-D_{1} z}-R_{10} e^{-S c z}+R_{11} e^{-D_{4} z}+\in e^{-n t}\left(R_{17} e^{-D_{1} z}+R_{18} e^{-D_{2} z}-R_{19} e^{-D_{3} z}\right. \\
\left.+R_{20} e^{-D_{4} z}-R_{21} e^{-S c z}+R_{22} e^{-D_{5} z}\right)
\end{gathered}
$$

Using $\mathrm{q}=\mathrm{u}+\mathrm{iv}$, the expressions for the primary and secondary velocities are obtained as follows

$$
\begin{aligned}
\mathrm{u}(\mathrm{z}, \mathrm{t})= & {\left[\left\{\left(\mathrm{K}_{9}+\mathrm{K}_{19} \in \mathrm{e}^{-\mathrm{nt}}\right) \cos \mathrm{A}_{2} \mathrm{z}+\left(\mathrm{K}_{10}+\mathrm{K}_{20} \in \mathrm{e}^{-\mathrm{nt}}\right) \sin \mathrm{A}_{2} \mathrm{z}\right\} \mathrm{e}^{-\mathrm{A}_{1} \mathrm{z}}\right.} \\
& +\left(\mathrm{K}_{25} \cos \mathrm{A}_{4} \mathrm{z}+\mathrm{K}_{26} \sin \mathrm{A}_{4} \mathrm{z}\right) \in \mathrm{e}^{-\mathrm{nt}} \mathrm{e}^{-\mathrm{A}_{3} \mathrm{z}}-\left(\mathrm{K}_{1}-\mathrm{K}_{13} \in \mathrm{e}^{-n \mathrm{nt}}\right) \mathrm{e}^{-\mathrm{D}_{1} \mathrm{z}} \\
& \left.+\mathrm{K}_{15} \in \mathrm{e}^{-\mathrm{nt}} \mathrm{e}^{-\mathrm{D}_{2} \mathrm{z}}-\mathrm{K}_{17} \in \mathrm{e}^{-\mathrm{nt}} \mathrm{e}^{-\mathrm{D}_{3} \mathrm{z}}-\left(\mathrm{K}_{3}+\mathrm{K}_{21} \in \mathrm{e}^{-\mathrm{nt}}\right) \mathrm{e}^{-\mathrm{Sc} \mathrm{z}}\right] \\
\mathrm{v}(\mathrm{z}, \mathrm{t})= & {\left[\left\{\left(\mathrm{K}_{10}+\mathrm{K}_{20} \in \mathrm{e}^{-\mathrm{nt}}\right) \cos \mathrm{A}_{2} \mathrm{z}-\left(\mathrm{K}_{9}+\mathrm{K}_{19} \in \mathrm{e}^{-\mathrm{nt}}\right) \sin \mathrm{A}_{2} \mathrm{z}\right\} \mathrm{e}^{-\mathrm{A}_{1} \mathrm{z}}\right.} \\
& +\left(-\mathrm{K}_{25} \sin \mathrm{A}_{4} \mathrm{z}+\mathrm{K}_{26} \cos \mathrm{A}_{4} \mathrm{z}\right) \in \mathrm{e}^{-\mathrm{nt}} \mathrm{e}^{-\mathrm{A}_{3} \mathrm{z}}-\left(\mathrm{K}_{2}-\mathrm{K}_{14} \in \mathrm{e}^{-\mathrm{nt}}\right) \mathrm{e}^{-\mathrm{D}_{1} \mathrm{z}} \\
& \left.+\mathrm{K}_{16} \in \mathrm{e}^{-\mathrm{nt}} \mathrm{e}^{-\mathrm{D}_{2} \mathrm{z}}-\mathrm{K}_{18} \in \mathrm{e}^{-\mathrm{nt}} \mathrm{e}^{-\mathrm{D}_{3} \mathrm{z}}-\left(\mathrm{K}_{4}+\mathrm{K}_{22} \in \mathrm{e}^{-\mathrm{nt}}\right) \mathrm{e}^{-\mathrm{Sc} \mathrm{z}}\right]
\end{aligned}
$$

\section{Skin Friction And Heat Transfer}

Once the expressions for velocities and temperature are known it is important to calculate the skinfriction and Nusselt number. The skin friction $\tau_{\mathrm{p}}$ is due to primary velocity $\mathrm{u}$ and skin friction $\tau_{\mathrm{s}}$ is due to secondary velocity $\mathrm{v}$ in the $\mathrm{x}$ and $\mathrm{y}$ directions respectively.

The coefficient of skin friction in $\mathrm{x}$ and $\mathrm{y}$ directions are:

$$
\begin{aligned}
& \tau_{\mathrm{x}}=\left(\frac{\partial \mathrm{u}}{\partial \mathrm{z}}\right)_{\mathrm{z}=0}=\left[\left\{-\left(\mathrm{k}_{9}+\mathrm{k}_{19} \in \mathrm{e}^{-\mathrm{nt}}\right) \mathrm{A}_{1}+\left(\mathrm{k}_{10}+\mathrm{k}_{20} \in \mathrm{e}^{-\mathrm{nt}}\right) \mathrm{A}_{2}\right\}\right. \\
& \left.-\left\{\mathrm{k}_{25} \mathrm{~A}_{3}-\mathrm{k}_{26} \mathrm{~A}_{4}+\mathrm{D}_{1} \mathrm{k}_{13}+\mathrm{D}_{2} \mathrm{k}_{15}-\mathrm{D}_{3} \mathrm{k}_{17}-\mathrm{Sc} \mathrm{k}_{21}\right\} \in \mathrm{e}^{-\mathrm{nt}}+\mathrm{D}_{1} \mathrm{k}_{1}+\mathrm{Sc} \mathrm{k}_{3}\right] \\
& \tau_{\mathrm{y}}=\left(\frac{\partial \mathrm{v}}{\partial \mathrm{z}}\right)_{\mathrm{z}=0}=\left[\left\{-\left(\mathrm{k}_{10}+\mathrm{k}_{20} \in \mathrm{e}^{-\mathrm{nt}}\right) \mathrm{A}_{1}-\left(\mathrm{k}_{9}+\mathrm{k}_{19} \in \mathrm{e}^{-\mathrm{nt}}\right) \mathrm{A}_{2}\right\}\right.
\end{aligned}
$$




$$
\left.\left.-\left\{\mathrm{k}_{26} \mathrm{~A}_{3}+\mathrm{k}_{25} \mathrm{~A}_{4}+\mathrm{D}_{1} \mathrm{k}_{14}+\mathrm{D}_{2} \mathrm{k}_{16}-\mathrm{D}_{3} \mathrm{k}_{18}-\mathrm{Sc} \mathrm{k}_{22}\right) \in \mathrm{e}^{-\mathrm{nt}}+\mathrm{D}_{1} \mathrm{k}_{2}+\mathrm{Sc} \mathrm{k}\right]_{4}\right]
$$

and the rate of heat transfer is given by

where

$$
\mathrm{Nu}=\frac{1}{\theta(0, \mathrm{t})}=\frac{\mathrm{D}_{1}}{1+\mathrm{D}_{1}\left(\mathrm{R}_{1}+\mathrm{R}_{2}\right) \in \mathrm{e}^{-\mathrm{nt}}}
$$

$$
\begin{aligned}
& \mathrm{A}_{1}=\frac{1+\mathrm{a}}{2}, \mathrm{~A}_{2}=\frac{\mathrm{b}}{2}, \mathrm{~A}_{3}=\frac{1+\mathrm{a}^{\prime}}{2}, \mathrm{~A}_{4}=\frac{\mathrm{b}^{\prime}}{2} \\
& \mathrm{a}=\left[\frac{\left\{\left(1+4 \mathrm{~K}^{-1}\right)^{2}+64 \mathrm{E}^{2}\right\}^{1 / 2}+\left(1+4 \mathrm{~K}^{-1}\right)}{2}\right]^{1 / 2} \\
& \mathrm{~b}=\left[\frac{\left\{\left(1+4 \mathrm{~K}^{-1}\right)^{2}+64 \mathrm{E}^{2}\right\}^{1 / 2}-\left(1+4 \mathrm{~K}^{-1}\right)}{2}\right]^{1 / 2}
\end{aligned}
$$

$$
a^{\prime}=\left[\frac{\left\{\left(1+4 K^{-1}+4 n\right)^{2}+64 E^{2}\right\}^{1 / 2}+\left(1+4 K^{-1}+4 n\right)}{2}\right]^{1 / 2}
$$

$$
\begin{aligned}
& \mathrm{b}^{\prime}=\left[\frac{\left\{\left(1+4 \mathrm{~K}^{-1}+4 \mathrm{n}\right)^{2}+64 \mathrm{E}^{2}\right\}^{1 / 2}-\left(1+4 \mathrm{~K}^{-1}+4 \mathrm{n}\right)}{2}\right]^{1 / 2} \\
& \mathrm{D}_{1}=\frac{\operatorname{Pr}+\sqrt{\mathrm{Pr}^{2}-4 \mathrm{~S}},}{2} \quad \mathrm{D}_{2}=\frac{\operatorname{Pr}+\sqrt{\operatorname{Pr}^{2}-4(\mathrm{n} \mathrm{Pr}+\mathrm{S})}}{2}, \quad \mathrm{D}_{4}=\frac{1+\sqrt{1+4\left(\mathrm{~K}^{-1}+2 \mathrm{iE}\right)}}{2}, \\
& \mathrm{D}_{3}=\frac{\mathrm{Sc}+\sqrt{\mathrm{Sc}^{2}-4 \mathrm{Sc}},}{2} \\
& \mathrm{D}_{5}=\frac{1+\sqrt{1+4\left(\mathrm{~K}^{-1}+\mathrm{n}+2 \mathrm{iE}\right)}}{2}
\end{aligned}
$$$$
\mathrm{D}_{2}=\frac{\operatorname{Pr}+\sqrt{\operatorname{Pr}^{2}-4(\mathrm{n} \operatorname{Pr}+\mathrm{S})}}{2},
$$

$$
\mathrm{D}_{4}=\frac{1+\sqrt{1+4\left(\mathrm{~K}^{-1}+2 \mathrm{iE}\right)}}{2} \text {, }
$$

$$
\mathrm{R}_{1}=\frac{\mathrm{Pr}}{\mathrm{D}_{1}^{2}-\operatorname{Pr} \mathrm{D}_{1}+(\mathrm{n} \operatorname{Pr}+\mathrm{S})}, \quad \mathrm{R}_{2}=-\frac{\mathrm{R}_{1} \mathrm{D}_{1}}{\mathrm{D}_{2}}
$$

$\mathrm{R}_{3}=\frac{\mathrm{D}_{1}(1+\mathrm{A})-\mathrm{Sc}}{\mathrm{Sc}\left(\mathrm{D}_{1}-\mathrm{Sc}\right)}$

$$
\mathrm{R}_{4}=\frac{\mathrm{A}}{\mathrm{D}_{1}-\mathrm{Sc}}
$$

$\mathrm{R}_{5}=\frac{\mathrm{R}_{3} \mathrm{Sc}}{\mathrm{n}}$

$$
\mathrm{R}_{6}=\frac{\mathrm{R}_{4} \mathrm{D}_{1} \mathrm{Sc}+\mathrm{AR}_{1} \mathrm{D}_{1}^{2}}{\mathrm{D}_{1}^{2}-\mathrm{ScD}_{1}+\mathrm{nSc}}
$$

$$
\begin{aligned}
& \mathrm{R}_{7}=\frac{\mathrm{AR}_{2} \mathrm{D}_{2}^{2}}{\mathrm{D}_{2}^{2}-\mathrm{Sc}_{2}+\mathrm{n} \mathrm{Sc}}, \quad \mathrm{R}_{8}=-\frac{\mathrm{R}_{5} \mathrm{Sc}+\mathrm{R}_{6} \mathrm{D}_{1}+\mathrm{R}_{7} \mathrm{D}_{2}}{\mathrm{D}_{3}}, \\
& \mathrm{R}_{9}=\mathrm{K}_{1}+\mathrm{iK}_{2}, \mathrm{R}_{10}=\mathrm{K}_{3}+\mathrm{i} \mathrm{K}_{4}, \mathrm{R}_{11}=\mathrm{K}_{9}+\mathrm{iK}_{10}, \\
& \mathrm{R}_{12}=\mathrm{K}_{5}+\mathrm{iK}_{6}, \mathrm{R}_{13}=-\mathrm{Gr} \mathrm{R}_{2}+\mathrm{Gm} \mathrm{\textrm {R } _ { 7 }}, \mathrm{R}_{14}=\mathrm{Gm} \mathrm{\textrm {R } _ { 8 } ,}
\end{aligned}
$$




$$
\begin{aligned}
& \mathrm{R}_{15}=\mathrm{K}_{11}+\mathrm{iK}_{12}, \mathrm{R}_{16}=\mathrm{K}_{7}+\mathrm{iK}_{8}, \mathrm{R}_{17}=\mathrm{K}_{13}+\mathrm{iK}_{14}, \mathrm{R}_{18}=\mathrm{K}_{15}+\mathrm{iK}_{16}, \\
& \mathrm{R}_{19}=\mathrm{K}_{17}+\mathrm{iK}_{18}, \mathrm{R}_{20}=\mathrm{K}_{19}+\mathrm{iK}_{20}, \mathrm{R}_{21}=\mathrm{K}_{21}+\mathrm{i} \mathrm{K}_{22}, \mathrm{R}_{22}=\mathrm{K}_{25}+\mathrm{iK}_{26}, \\
& \mathrm{~K}_{1}=\frac{\left(\mathrm{Gr}-\mathrm{D}_{1} \mathrm{Gm} \mathrm{R}_{4}\right)\left(\mathrm{D}_{1}^{2}-\mathrm{D}_{1}-\mathrm{K}^{-1}\right)}{\mathrm{D}_{1}^{2}\left\{\left(\mathrm{D}_{1}^{2}-\mathrm{D}_{1}-\mathrm{K}^{-1}\right)^{2}+4 \mathrm{E}^{2}\right\}}, \mathrm{K}_{2}=\frac{2 \mathrm{E}\left(\mathrm{Gr}-\mathrm{D}_{1} \mathrm{Gm} \mathrm{R} \mathrm{R}_{4}\right)}{\mathrm{D}_{1}^{2}\left\{\left(\mathrm{D}_{1}^{2}-\mathrm{D}_{1}-\mathrm{K}^{-1}\right)^{2}+4 \mathrm{E}^{2}\right\}}, \\
& \mathrm{K}_{3}=\frac{\left(\mathrm{Sc}^{2}-\mathrm{Sc}-\mathrm{K}^{-1}\right) \mathrm{Gm} \mathrm{R} \mathrm{R}_{3}}{\left(\mathrm{Sc}^{2}-\mathrm{Sc}-\mathrm{K}^{-1}\right)^{2}+4 \mathrm{E}^{2}}, \quad \mathrm{~K}_{4}=\frac{2 \mathrm{E} \mathrm{Gm} \mathrm{R_{3 }}}{\left(\mathrm{Sc}^{2}-\mathrm{Sc}-\mathrm{K}^{-1}\right)^{2}+4 \mathrm{E}^{2}},
\end{aligned}
$$$$
\mathrm{K}_{4}=\frac{2 \mathrm{E} \mathrm{Gm} \mathrm{R}}{\left(\mathrm{Sc}^{2}-\mathrm{Sc}-\mathrm{K}^{-1}\right)^{2}+4 \mathrm{E}^{2}} \text {, }
$$$$
\mathrm{K}_{5}=-\mathrm{Gr} \mathrm{R}_{1}+\mathrm{Gm} \mathrm{R} \mathrm{R}_{5}-\mathrm{K}_{1} \mathrm{D}_{1} \text {, }
$$$$
\mathrm{K}_{6}=-\mathrm{K}_{2} \mathrm{D}_{1} \text {, }
$$$$
\mathrm{K}_{7}=\mathrm{Gm} \mathrm{R}_{5}+\mathrm{Sc} \mathrm{\textrm {K } _ { 3 }} \text {, }
$$$$
\mathrm{K}_{8}=\mathrm{Sc} \mathrm{K}_{4} \text {, }
$$$$
\mathrm{K}_{9}=\left[\frac{\left(1+\mathrm{h} \mathrm{A}_{1}\right)\left[\mathrm{K}_{1}\left(1+\mathrm{h} \mathrm{D}_{1}\right)+\mathrm{K}_{3}(1+\mathrm{h} \mathrm{Sc})\right]+\mathrm{h} \mathrm{A}_{2}\left[\mathrm{~K}_{2}\left(1+\mathrm{hD}_{1}\right)+\mathrm{K}_{4}(1+\mathrm{h} \mathrm{Sc})\right]}{\left(1+\mathrm{h} \mathrm{A}_{1}\right)^{2}+\mathrm{h}^{2} \mathrm{~A}_{2}^{2}}\right] \text {, }
$$$$
\mathrm{K}_{10}=\left[\frac{\left(1+\mathrm{h} \mathrm{A}_{1}\right)\left[\mathrm{K}_{2}\left(1+\mathrm{h} \mathrm{D}_{1}\right)+\mathrm{K}_{4}(1+\mathrm{h} \mathrm{Sc})\right]-\mathrm{h} \mathrm{A}_{2}\left[\mathrm{~K}_{1}\left(1+\mathrm{h} \mathrm{D}_{1}\right)+\mathrm{K}_{3}(1+\mathrm{h} \mathrm{Sc})\right.}{\left(1+\mathrm{h} \mathrm{A}_{1}\right)^{2}+\mathrm{h}^{2} \mathrm{~A}_{2}^{2}}\right] \text {, }
$$

$$
\begin{aligned}
& \mathrm{K}_{11}=\mathrm{A}_{1} \mathrm{R}_{9}-\mathrm{A}_{2} \mathrm{~K}_{10}, \quad \mathrm{~K}_{12}=\mathrm{A}_{2} \mathrm{R}_{9}+\mathrm{A}_{1} \mathrm{~K}_{10}, \\
& \mathrm{~K}_{13}=\left[\frac{\mathrm{K}_{5}\left\{\left(\mathrm{D}_{1}^{2}-\mathrm{D}_{1}-\mathrm{n}-\mathrm{K}^{-1}\right)\right\}-2 \mathrm{E} \mathrm{K}_{6}}{\left(\mathrm{D}_{1}^{2}-\mathrm{D}_{1}-\mathrm{n}-\mathrm{K}^{-1}\right)^{2}+4 \mathrm{E}^{2}}\right],
\end{aligned}
$$$$
\mathrm{K}_{14}=\left[\frac{\mathrm{K}_{6}\left\{\left(\mathrm{D}_{1}^{2}-\mathrm{D}_{1}-\mathrm{n}-\mathrm{K}^{-1}\right)+2 \mathrm{E} \mathrm{K}_{5}\right.}{\left(\mathrm{D}_{1}^{2}-\mathrm{D}_{1}-\mathrm{n}-\mathrm{K}^{-1}\right)^{2}+4 \mathrm{E}^{2}}\right] \text {, }
$$$$
\mathrm{K}_{15}=\frac{\mathrm{R}_{13}\left(\mathrm{D}_{2}^{2}-\mathrm{D}_{2}-\mathrm{n}-\mathrm{K}^{-1}\right)}{\left(\mathrm{D}_{2}^{2}-\mathrm{D}_{2}-\mathrm{n}-\mathrm{K}^{-1}\right)^{2}+4 \mathrm{E}^{2}}, \quad \mathrm{~K}_{16}=\frac{2 \mathrm{E} \mathrm{R}}{\left(\mathrm{D}_{2}^{2}-\mathrm{D}_{2}-\mathrm{n}-\mathrm{K}^{-1}\right)^{2}+4 \mathrm{E}^{2}},
$$$$
K_{17}=\frac{R_{14}\left(D_{3}^{2}-D_{3}-n-K^{-1}\right)}{\left(D_{3}^{2}-D_{3}-n-K^{-1}\right)^{2}+4 E^{2}}, \quad K_{18}=\frac{2 \mathrm{E} \mathrm{R}_{14}}{\left(D_{3}^{2}-D_{3}-n-K^{-1}\right)^{2}+4 E^{2}},
$$$$
\mathrm{K}_{19}=\left[\frac{\mathrm{K}_{11}\left(\mathrm{~A}_{1}^{2}-\mathrm{A}_{2}^{2}-\mathrm{A}_{1}-\mathrm{n}-\mathrm{K}^{-1}\right)+\mathrm{K}_{12}\left(2 \mathrm{~A}_{1} \mathrm{~A}_{2}-\mathrm{A}_{2}-2 \mathrm{E}\right)}{\left(\mathrm{A}_{1}^{2}-\mathrm{A}_{2}^{2}-\mathrm{A}_{1}-\mathrm{n}-\mathrm{K}^{-1}\right)^{2}+\left(2 \mathrm{~A}_{1} \mathrm{~A}_{2}-\mathrm{A}_{2}-2 \mathrm{E}\right)^{2}}\right] \text {, }
$$$$
K_{20}=\left[\frac{K_{12}\left(A_{1}^{2}-A_{2}^{2}-A_{1}-n-K^{-1}\right)-K_{11}\left(2 A_{1} A_{2}-A_{2}-2 E\right)}{\left(A_{1}^{2}-A_{2}^{2}-A_{1}-n-K^{-1}\right)^{2}+\left(2 A_{1} A_{2}-A_{2}-2 E\right)^{2}}\right] \text {, }
$$ 


$$
\begin{aligned}
\mathrm{K}_{21}=\left[\frac{\mathrm{K}_{7}\left(\mathrm{Sc}^{2}-\mathrm{Sc}-\mathrm{n}-\mathrm{K}^{-1}\right)-2 \mathrm{E} \mathrm{K}_{8}}{\left(\mathrm{Sc}^{2}-\mathrm{Sc}-\mathrm{n}-\mathrm{K}^{-1}\right)+4 \mathrm{E}^{2}}\right], \\
\mathrm{K}_{22}=\left[\frac{\mathrm{K}_{8}\left(\mathrm{Sc}^{2}-\mathrm{Sc}-\mathrm{n}-\mathrm{K}^{-1}\right)+2 \mathrm{EK}_{7}}{\left(\mathrm{Sc}^{2}-\mathrm{Sc}-\mathrm{n}-\mathrm{K}^{-1}\right)^{2}+4 \mathrm{E}^{2}}\right], \\
\mathrm{K}_{23}=\left[-\mathrm{K}_{13}\left(1+\mathrm{hD}_{1}\right)-\mathrm{K}_{15}\left(1+\mathrm{hD}_{2}\right)+\mathrm{K}_{17}\left(1+\mathrm{hD}_{3}\right)-\mathrm{K}_{19}(1+\mathrm{hA})\right. \\
\left.\quad+\mathrm{K}_{20} \mathrm{hA}_{2}+\mathrm{K}_{21}(1+\mathrm{hSc})\right], \\
\mathrm{K}_{24}=\left[\mathrm{K}_{14}\left(1+\mathrm{hD}_{1}\right)+\mathrm{K}_{16}\left(1+\mathrm{hD}_{2}\right)-\mathrm{K}_{18}\left(1+\mathrm{hD}_{2}\right)-\mathrm{K}_{22}(1+\mathrm{hSc})\right], \\
\mathrm{K}_{25}=\left[\frac{\mathrm{K}_{23}\left(1+\mathrm{hA}_{3}\right)+\mathrm{K}_{24} \mathrm{hA}_{4}}{\left(1+\mathrm{hA}_{3}\right)^{2}+\mathrm{h}^{2} \mathrm{~A}_{4}^{2}}\right],
\end{aligned}
$$

\section{Discussion And Conclusions}

In order to get physical insight of the problem, calculations have been made for primary and secondary velocities $(\mathrm{u}, \mathrm{v})$, temperature $(\theta)$, concentration $(\phi)$, primary and secondary skin-frictions $\left(\tau_{\mathrm{p}}, \tau_{\mathrm{s}}\right)$ and Nusselt number $(\mathrm{Nu})$ for different parameters viz. Prandtl number (Pr), Schmidt Number $(\mathrm{Sc})$, Permeability parameter (K). Heat source parameter (S), Grashof number for heat transfer (Gr), Grashof number for mass transfer $(\mathrm{Gm})$, Diffusion parameter (A) and Slip parameter (h) fixing $\in=0.02, \mathrm{E}=0.4, \mathrm{n}=0.1$ and $\mathrm{t}=1.0$.

From Figure 1, important observation is that increase in h, increases the primary velocity near the plate but after some distance it decreases Physically it due to the fact that effect of slip parameter nullify as we go far from the plate. However, in case of $\mathrm{Gm}$ and $\mathrm{S}$ velocity increases as both the parameters decreases but $\mathrm{Gr}$ has opposite phenomena. From Figure 2, for secondary velocity v (in the figure we have plotted $-\mathrm{v}$ ) the observations are same as that of primary velocity for the case of $\mathrm{Gr}, \mathrm{Gm}$ and $\mathrm{S}$ but interestingly as $\mathrm{h}$ increases secondary velocity decrease near the plate but goes on increasing as we move away from the plate.

In figure 3 and 4 the primary and secondary velocities $\mathrm{u}$ and $\mathrm{v}$ are plotted for different values of $\mathrm{Pr}, \mathrm{K}$ and Sc. From the figures we observed that an increase in Pr and Sc decreases primary but increases secondary velocity. It is further observed that for increasing $\mathrm{K}$ both velocities $(\mathrm{u}, \mathrm{v})$ increases. Physically, we say that increase in $\mathrm{K}$ increases the flow space in the porous medium and hence both velocities increase $\quad(K=\infty$ is free flow).

The non-dimensional temperature $\theta$ for different values of $\mathrm{S}$ and $\operatorname{Pr}$ is shown in figure 5. From this figure it is concluded that an increase in $\operatorname{Pr}$ and $\mathrm{S}$ temperature decreases for both the basic fluids air $(\operatorname{Pr}=0.71)$ and water $(\operatorname{Pr}=7.0)$. It is interesting to note that temperature is always move for air $(\operatorname{Pr}=0.71)$ as compared to water $\quad(\operatorname{Pr}=7.0)$ for every value of $\mathrm{S}$. Moreover, for both the fluids temperature is move when heat is observed by the fluid $(-\mathrm{S})$.

The concentration profile $\phi$ is shown in Figure 6 against $\mathrm{z}$ for different values of A, Pr and Sc. It is concluded that increase in $\operatorname{Pr}$ and Sc decreases concentration but for diffusion parameter A phenomena is opposite in nature.

Primary and secondary skin friction $\tau_{\mathrm{p}}$ and $\tau_{\mathrm{s}}$ plotted against $\mathrm{S}$ are shown in figures 7 and 8 for different values of $\mathrm{h}, \mathrm{Gr}$. Gm, Pr, K and Sc. It is noted that the primary skin friction at the plate decreases with the increase in $\mathrm{h}, \mathrm{Pr}$ and Sc but increases with increase in $\mathrm{K}, \mathrm{Gr}$ and $\mathrm{Gm}$.

For secondary skin friction at the plate, it is observed that it increases with the increase in $\mathrm{Gr}, \mathrm{Gm}, \mathrm{K}$ while decreases with the increase in $\mathrm{Sc}, \mathrm{h}$ and Pr. Nusselt number $(\mathrm{Nu})$ is shown in Figure 9, plotted against $\mathrm{S}$ for the same fixed values of $\in, \mathrm{n}$

And t. From the figure we observe that increase in Pr decreases the Nusselt number hence Nusselt number is more for air than water. 


\section{REFERENCES}

[1]. V. M. Soundalgekar, N. V.Vighnesam and I. Pop, Combined free and forced convection flow past a vertical porous plate, Int. J. Energy Research 5, 1981, 215-226.

[2]. A. Raptis and C. Predikis, Mass transfer and free convection flow through a porous medium, Int. J. Energy Research, 11 1987, 423428.

[3]. A. S. Mohammad, Free and forced convection boundary layer flow through a porous medium with large suction", Int. J. Energy Research, 17 (1993), 1-7.

[4]. K. A. Helmy, MHD unsteady free convection flow past a vertical porous plate, ZAMM, J. applied mathematics and mechanics, 78 1998, 255-270.

[5]. N. C. Jain and P.Gupta, Hall effects on unsteady free convection slip flow in a rotating fluid along a porous vertical surface bounded by porous medium, Int. J. Applied Mechanics and Engineering, 12 2007, 609-627.

[6]. B. C. Chandrasekhara and P.M.S. Namboodiri, Influence of variable permeability on combined free and forced convection about inclined surface in porous, Int. J. Heat mass transfer, 28 1985, 199-206.

[7]. Y. Joshi and B. Gebhart, Mixed convection in porous medium adjacent to a vertical uniform heat flux surface, Int. J. Heat mass transfer, 28 1985, 1783-1786.

[8]. F. C.Lai and F.A. Kulacki, The effect of variable viscosity on convective heat transfer along a vertical surface in a saturated porous medium, Int. J. Heat mass transfer, 33, 1990, 1028-1031.

[9]. A. Raptis and N.G. Kafousias, N.G., "Free convection and mass transfer flow through a porous medium under the action of magnetic field", Rev. Roun. Sci. Tech. Mec. Appl. 27 (1982), 37-43.

[10]. A. Bejan and K.R. Khair, Effect of thermal diffusion on an oscillatory MHD convective flow with mass transfer through a porou s medium. Int. J. Heat mass transfer, 28 1985, 902-918.

[11]. E. M. A. Elbashbeshy, Heat and mass transfer along a vertical plate in presence of a magnetic field, Ind. J. Pure. Appl. Math., 27 1996, 621-631.

[12]. M. Acharya, G.C. Dash and L.P. Singh, Magnetic field effects on the free convection and mass transfer flow through porous medium with constant suction and constant heat flux, Ind. J. Pure. Appl. Math., 31 2000, 1-18.

[13]. B. Chand, A. Kumar and V.P. Kaushik, Magnetic field effects on the free convection and mass transfer flow through porous medium with time dependent suction and constant heat flux, Bull. Cal. Math. Soc. 95 2003, 259-272.

[14]. H. S. Tsien, Superaerodynamics mechanics of rarefied gas, J. Aeronautical Sciences, 13 1946, 653-664.

[15]. R. E. Street, A study of boundary conditions in slip flow aerodynamics in rarefied gas dynamics, Pergamon Press 1960.

[16]. S. Jothimani, and Devi, Anjali, S.P., MHD Couette flow with heat transfer and slip flow effects in an inclined channel, Ind. J. Math. 43 2001, 47-62.

[17]. N. C. Jain, B. Sharma and D. Vijay, Three-dimensional free convection heat transfer flow with periodic permeability and periodic temperature in slip flow regime, J. Energy. Heat and mass transfer, 28, 2006, 29-44.

[18]. P. Gupta, B. Sharma and N. C. Jain, MHD heat and mass transfer slip flow in a vertical porous channel with variable suction and heat source, Mod. Mes. Control B (Modelling, Measurement and Control B), 78 2009, 34-46.

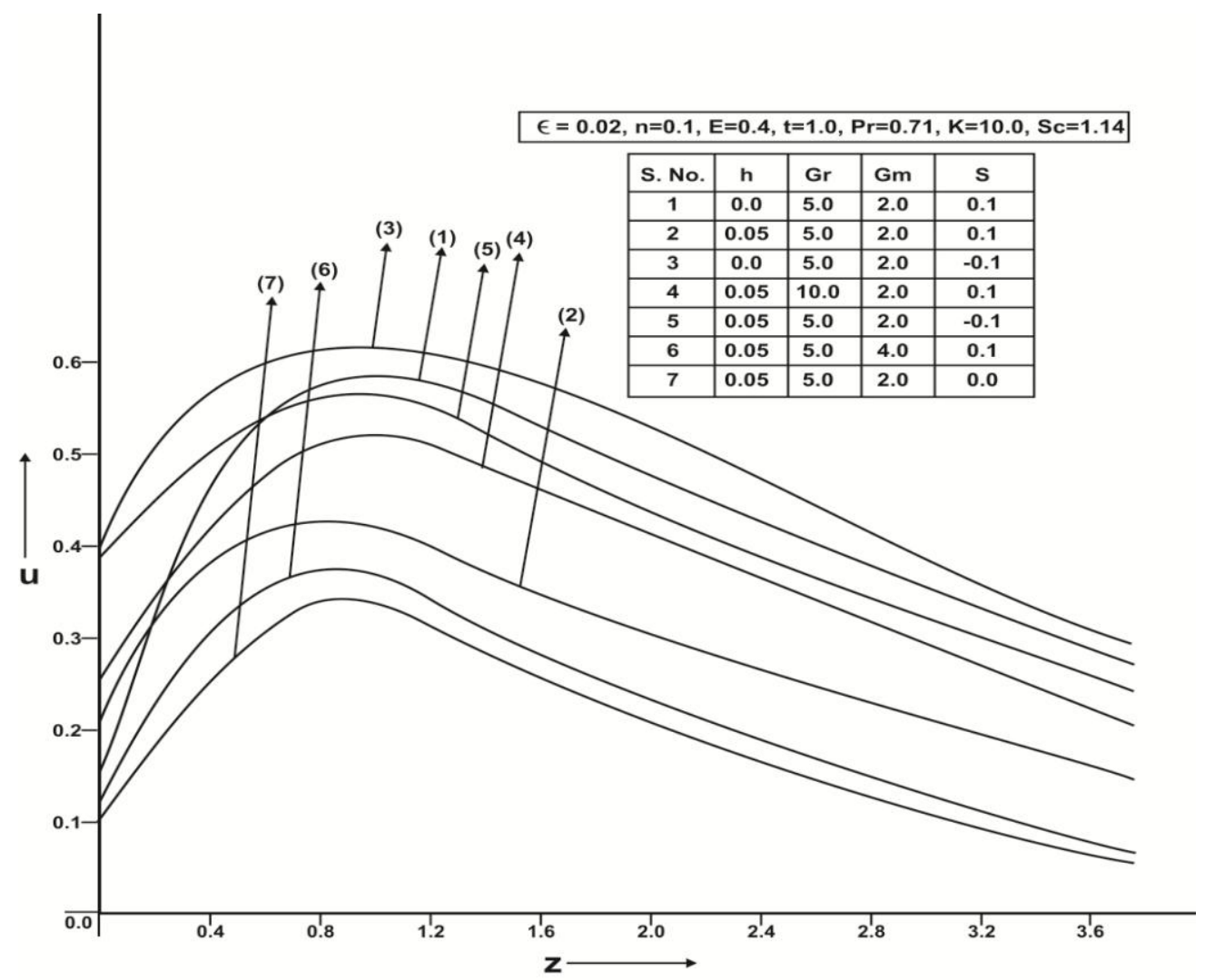

Figure 1. : Primary Velocity Distribution $\mathbf{U}$ plotted against $\mathbf{Z}$ for different values of $\mathrm{h}, \mathrm{Gr}, \mathrm{Gm}$ and $\mathrm{S}$ 


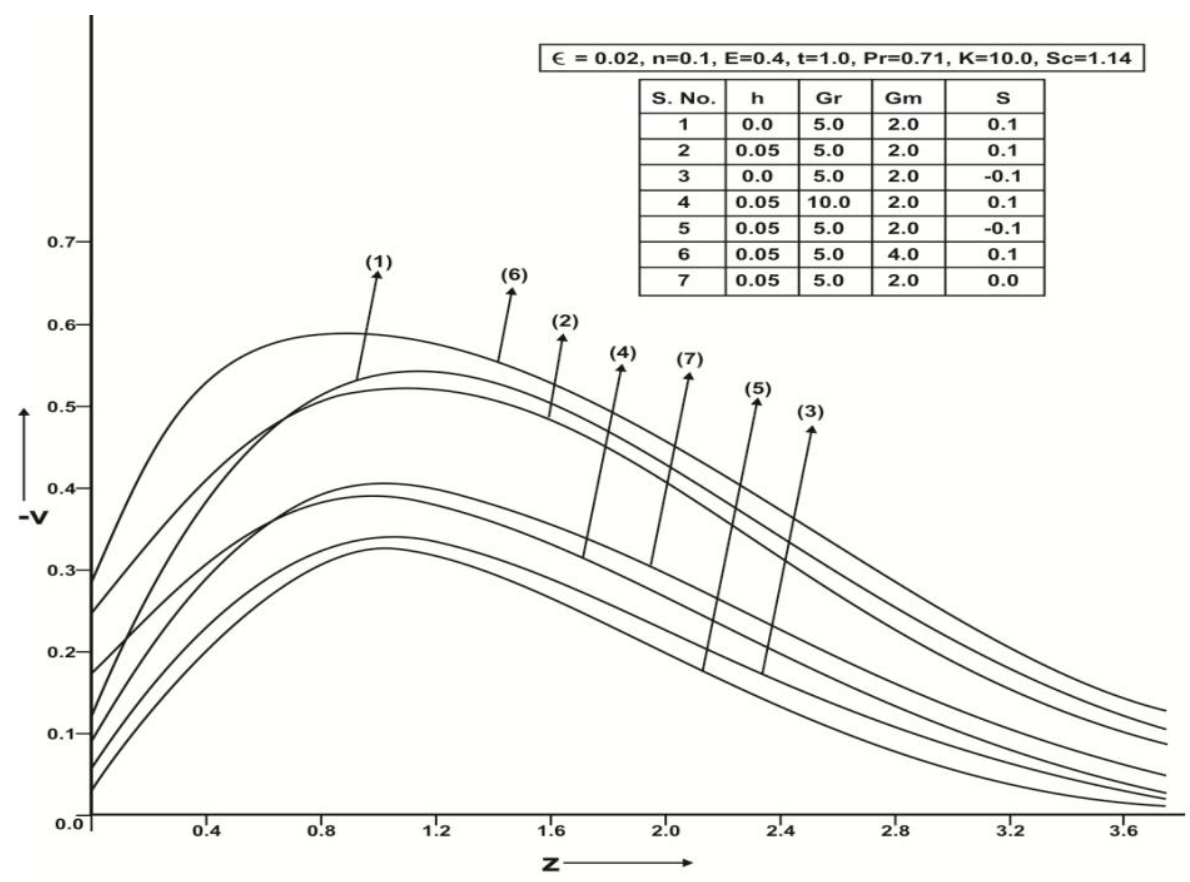

Figure 2. : Secondary Velocity Distribution $-\mathrm{V}$ plotted against $\mathbf{Z}$ for different values of $\mathrm{h}, \mathrm{Gr}, \mathrm{Gm}$ and $\mathrm{S}$

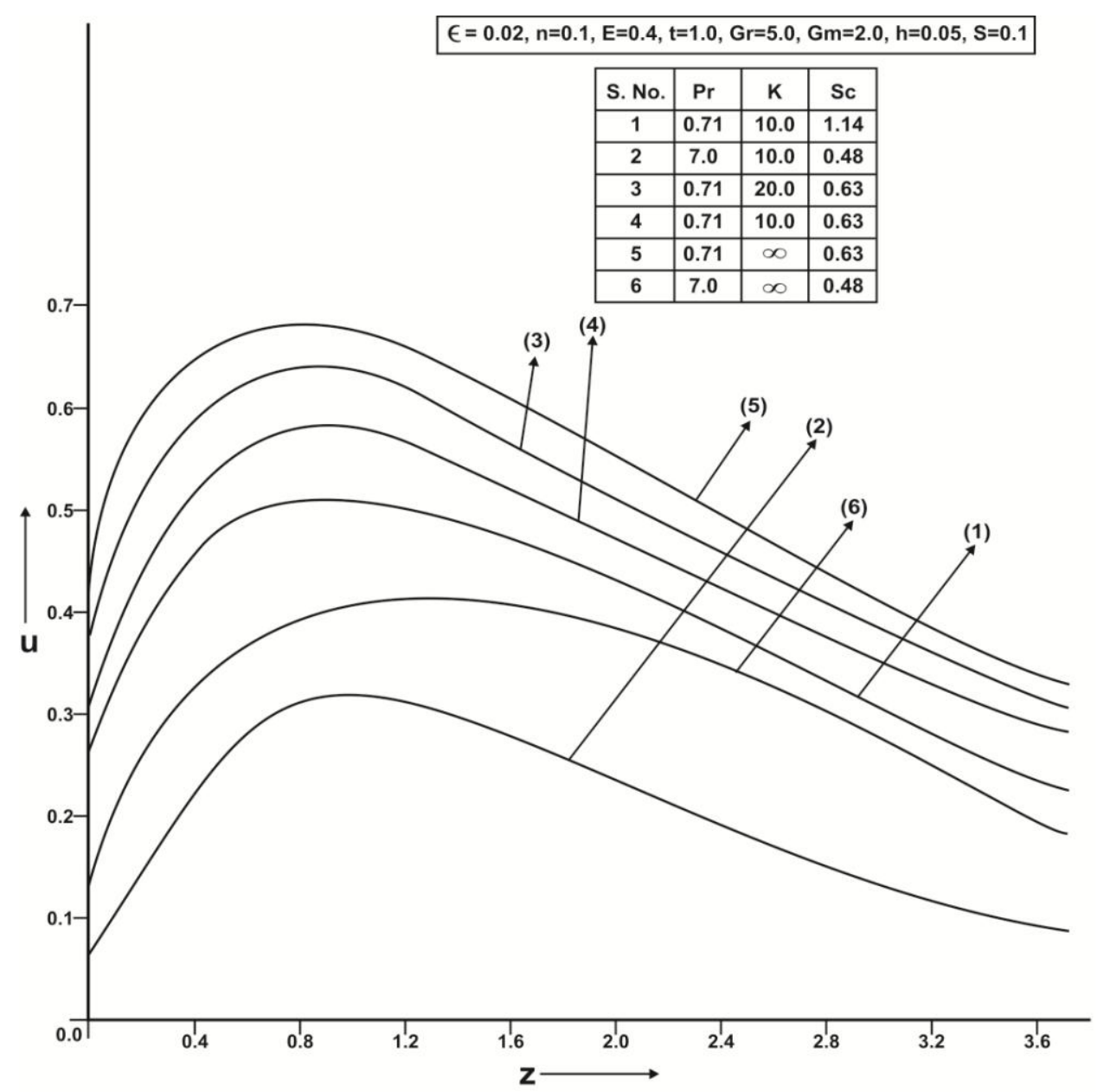

Figure 3. : Primary Velocity Distribution $\mathbf{U}$ plotted against $\mathbf{Z}$ for different values of $\mathrm{Pr}, \mathrm{K}$ and $\mathrm{Sc}$ 


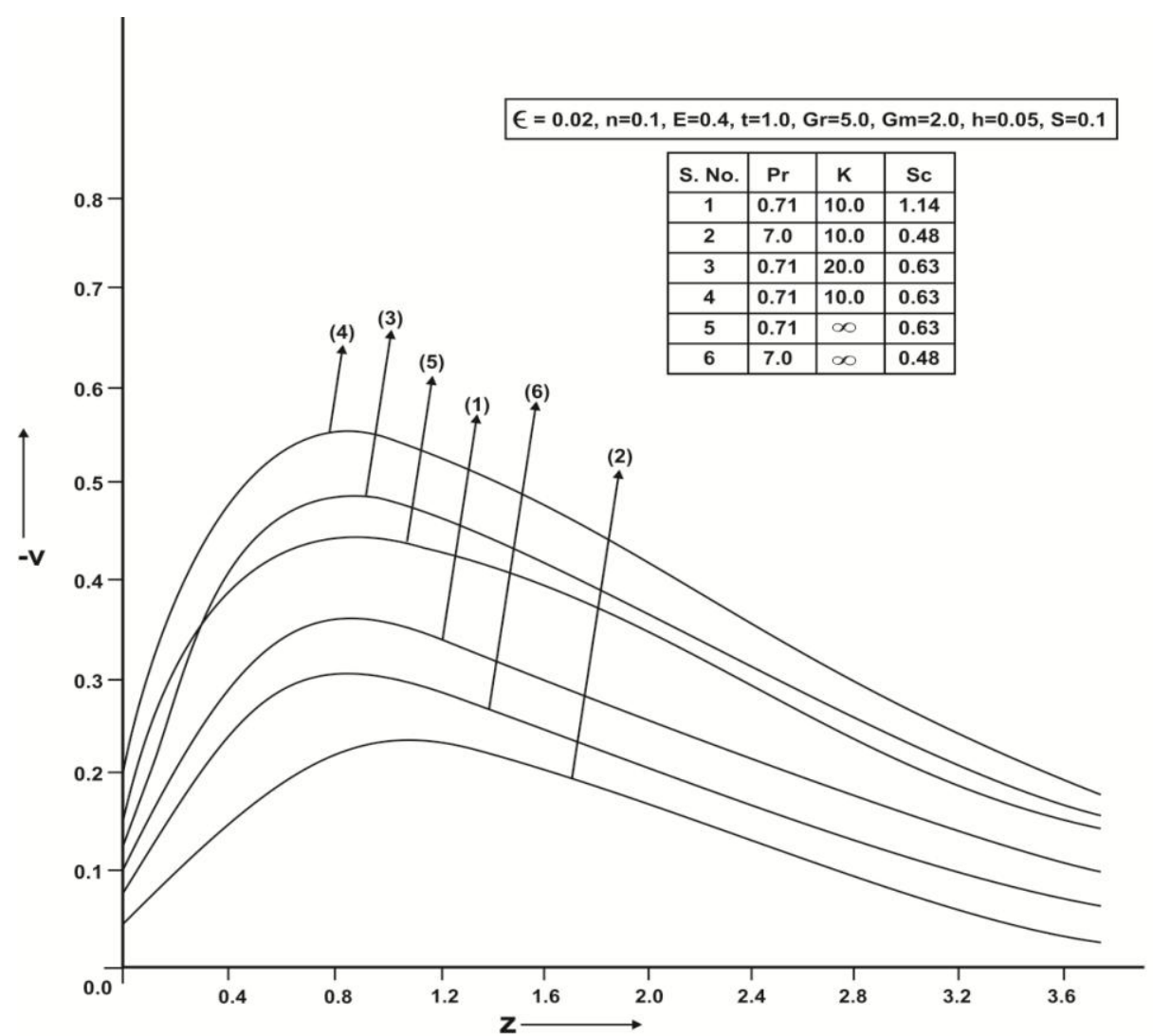

Figure 4. : Secondary Velocity Distribution $-\mathbf{V}$ plotted against $\mathbf{Z}$ for different values of $\mathrm{Pr}, \mathrm{K}$ and $\mathrm{Sc}$

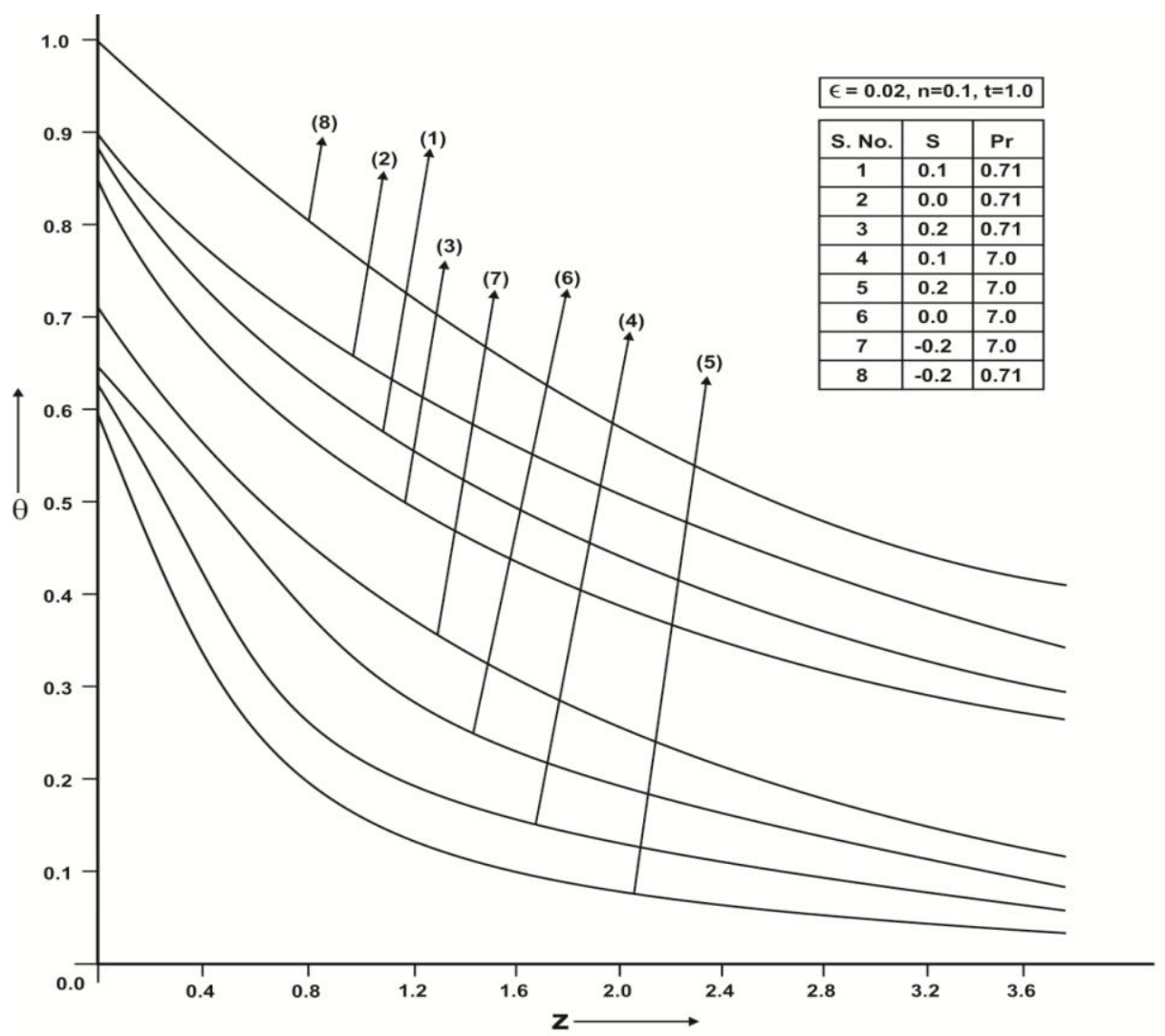

Figure 5. : Temperature Distribution $\theta$ plotted against $\mathbf{Z}$ for different values of $\mathbf{S}$ and $\mathbf{P r}$. 


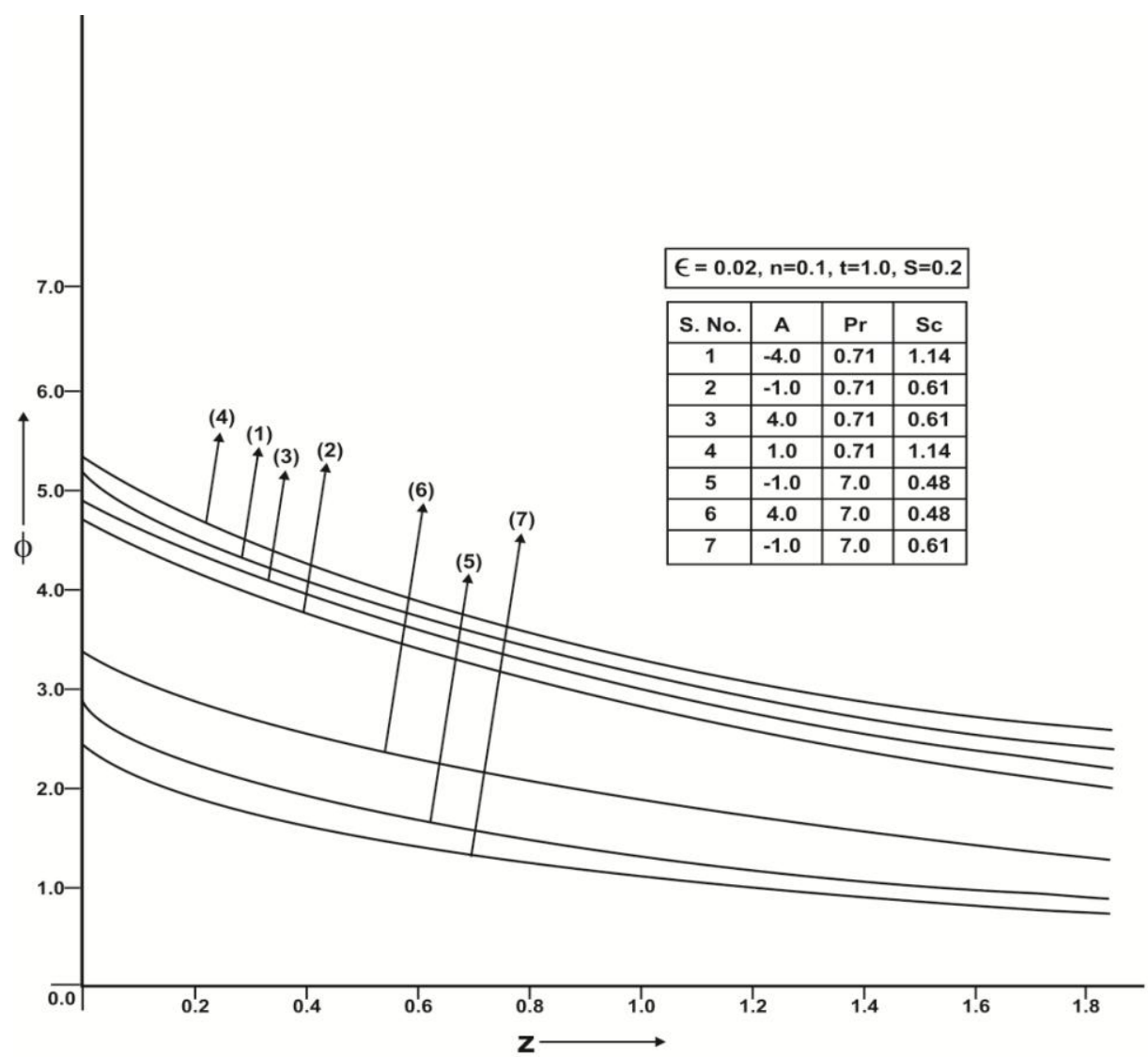

Figure 6. : Concentration Profile $\phi$ plotted against $\mathbf{Z}$ for different values of $\mathrm{A}, \mathrm{Pr}$ and $\mathrm{Sc}$.

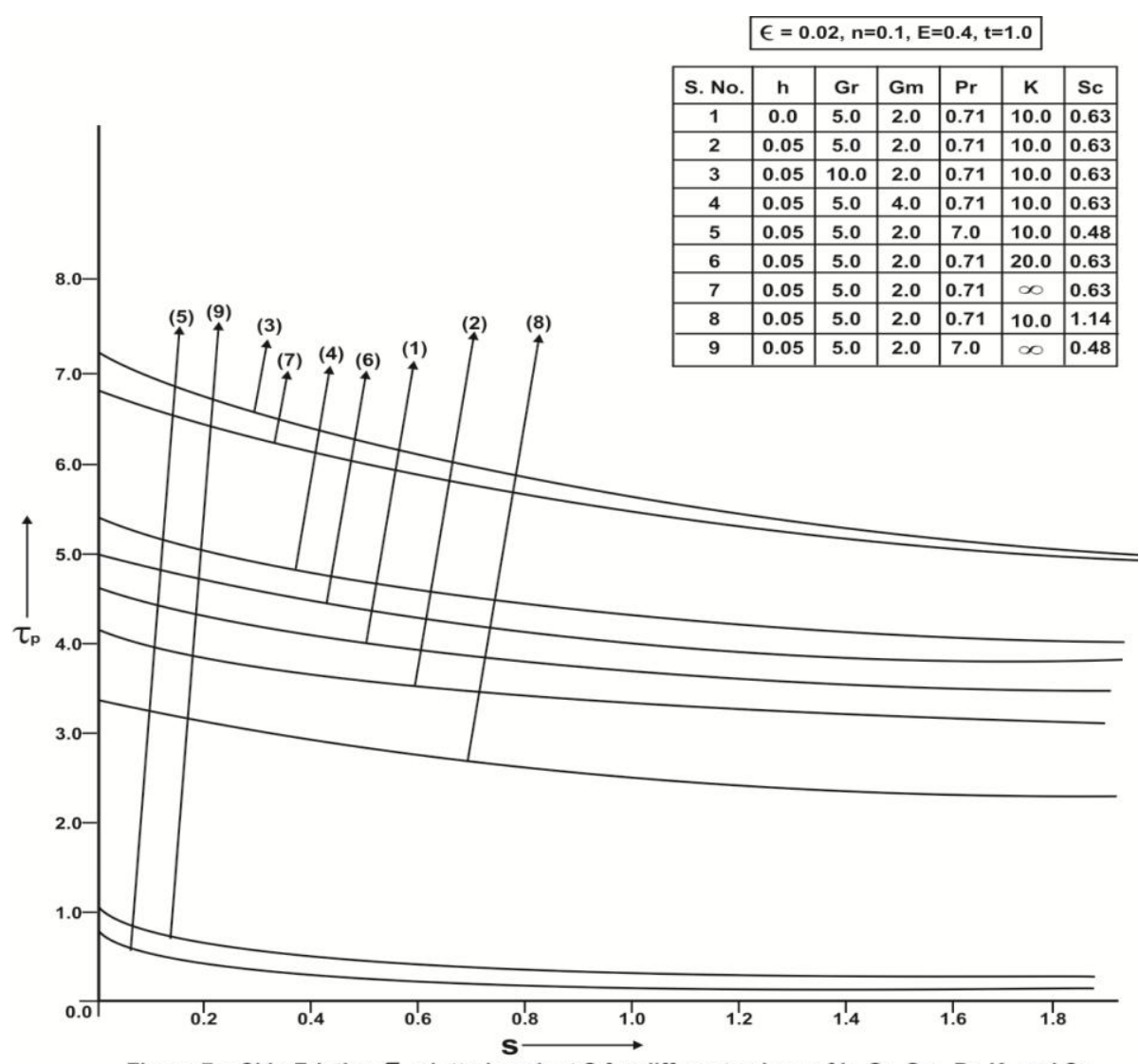

Figure 7. : Skin Friction $\tau_{\mathrm{p}}$ plotted against $\mathrm{S}$ for different values of $\mathrm{h}, \mathrm{Gr}, \mathrm{Gm}, \mathrm{Pr}, \mathrm{K}$, and $\mathrm{Sc}$ 


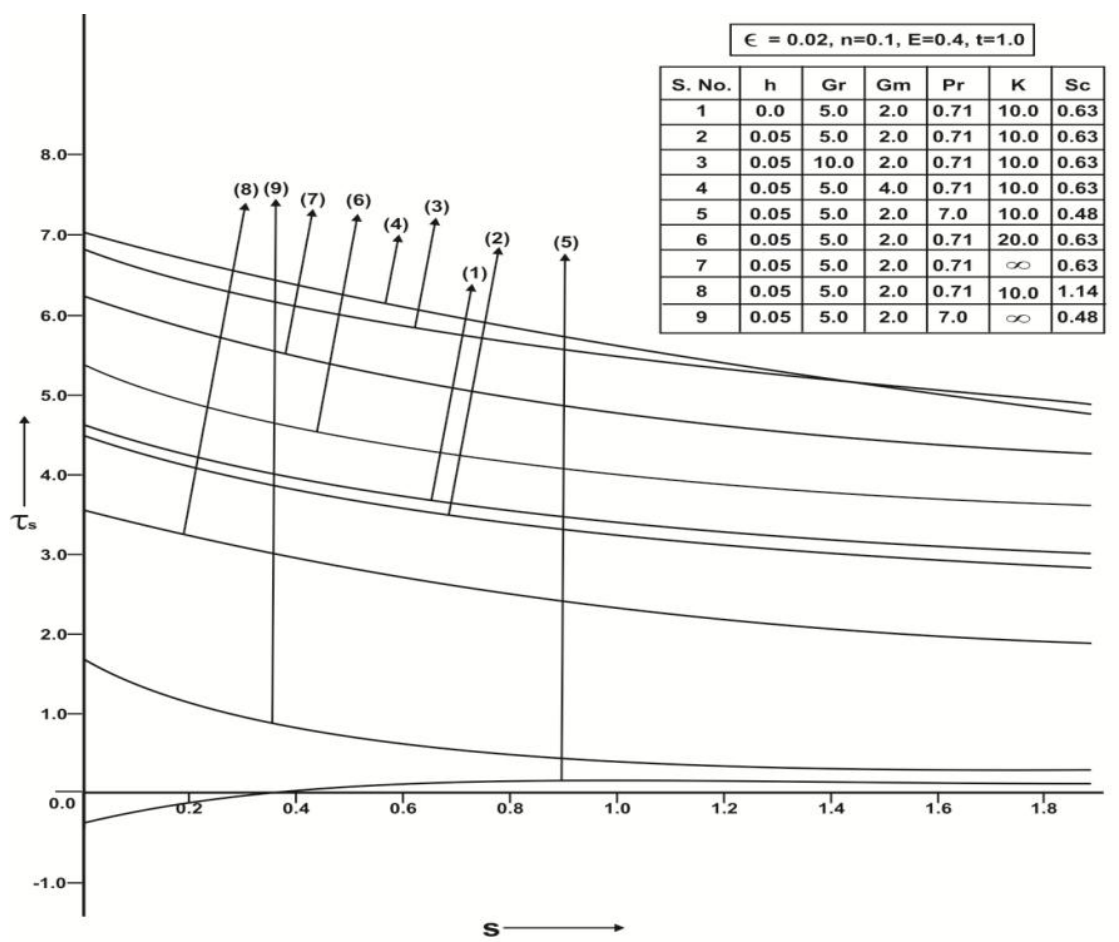

Figure 8. : Skin Friction $\tau_{\text {s }}$ plotted against $S$ for different values of $h, G r, G m, P r, K$, and Sc

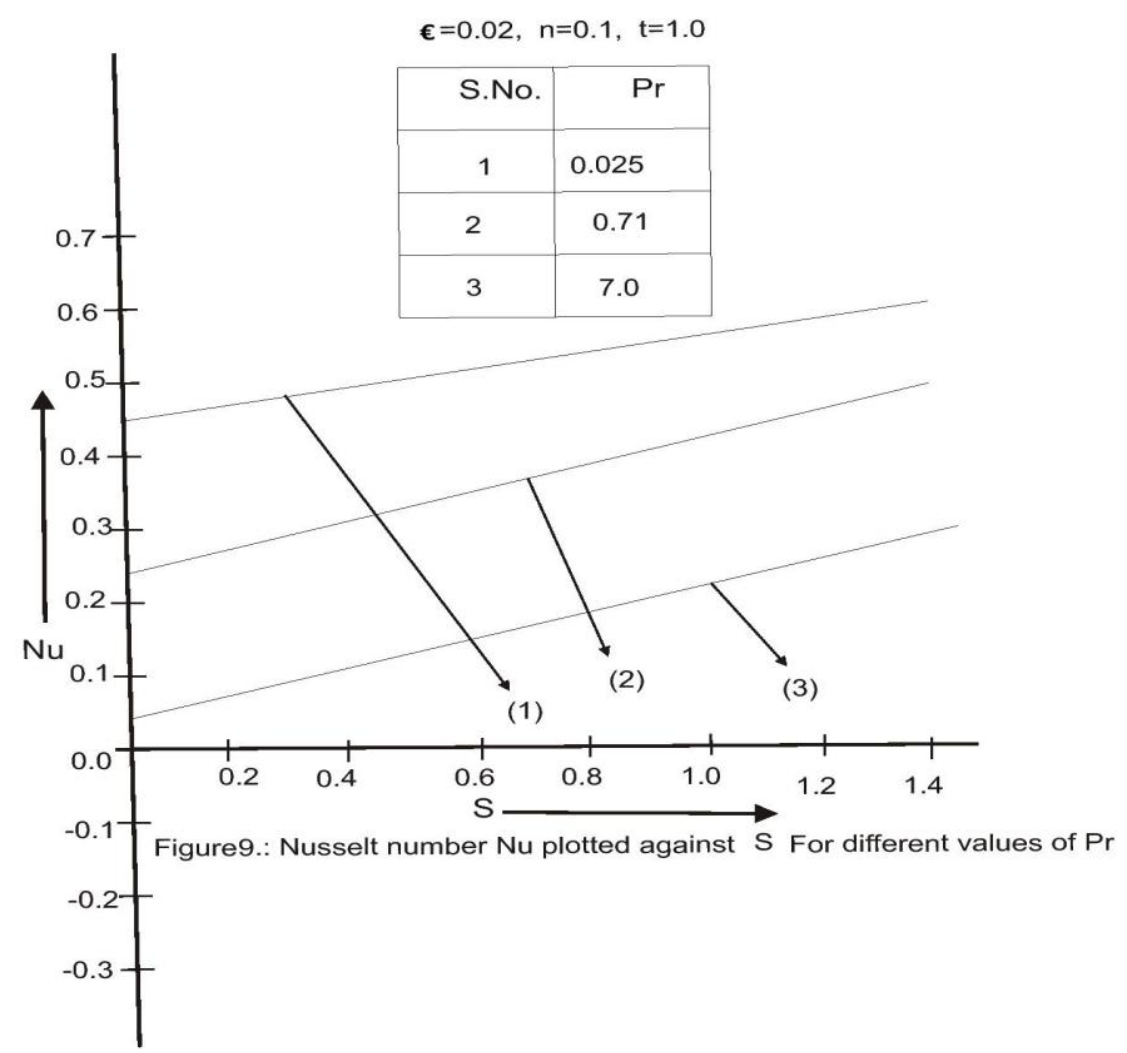

